\title{
Uncovering the pharmacological mechanism of strychnos nux-uomical in the treatment of peripheral neuropathy based on a network pharmacology approach
}

\section{JingJing Xiang}

Zhejiang Hospital of Traditional Chinese Medicine Department of Hematology

Chen-Jun Lin

Zhejiang Chinese Medical University

\section{Yu Zhang}

Zhejiang Hospital of Traditional Chinese Medicine Department of Hematology

Ni Zhu

Zhejiang Hospital of Traditional Chinese Medicine Department of Hematology Jian-Ping Shen

Zhejiang Hospital of Traditional Chinese Medicine Department of Hematology Jun-Fa Chen

Zhejiang Hospital of Traditional Chinese Medicine Department of Hematology Jian-You Gu

Zhejiang Hospital of Traditional Chinese Medicine Department of Hematology

\section{Yan Zhou}

Zhejiang Chinese Medical University

\section{Ke-Ting Jin}

Zhejiang Chinese Medical University

\section{Zi-Rui Hong}

Zhejiang Chinese Medical University

Shu Deng ( $\square$ dengshu2021@163.com )

Zhejiang Chinese Medical University https://orcid.org/0000-0001-8353-6705

\section{Research}

Keywords: Network pharmacology, Peripheral neuropathy, Strychnos nux-uomical, TCM systematic pharmacological analysis

Posted Date: July 13th, 2021 
DOl: https://doi.org/10.21203/rs.3.rs-684270/v1

License: (c) (1) This work is licensed under a Creative Commons Attribution 4.0 International License. Read Full License 


\section{Abstract}

Background: Strychnos nux-uomical (Ma Qianzi in pinyin), a classic chinese herb, has been extensively used to treat peripheral neuropathy in clinical practice in china for many years. However, the pharmacological mechanisms of strychnos nux-uomical remain vague. The aim of this study was to decrypt the underlying mechanisms of strychnos nux-uomical in the treatment of peripheral neuropathy using a systems harmacology approach.

Methods: Using pharmacokinetic parameters [oral bioavailability $(\mathrm{OB}) \geq 30 \%$ and drug likeness $(D L) \geq 0.18$ ] of traditional chinese medicine system pharmacology platform (TCMSP) to screen the active ingredient of strychnos nux-uomical, and then integrating target prediction human gene database to predict and screen its target genes for the treatment of strychnos nux-uomical, network topology attribute analysis software cytoscape 3.6.0 was utilized to construct the active components-peripheral neuropath targets network. String database and cytoscape software were used to draw the protein interaction network. Finally gene ontology (GO) and kyoto encyclopedia of genes and genomes (KEGG) pathway enrichment analyses by panther database.

Results: A total of 13 potential active ingredients were screened based on their OB and DL parameters, and 72 core targets were related to disease target. The results of pathway enrichment analysis indicated that putative targets of strychnos nux-uomical mostly participated in various pathways associated with cellular process, response to stimulus, biological regulation, metabolic process, localization. The putative targets of strychnos nux-uomical (endothelial growth factor, tumor necrosis factor-a, interleukin-10, interleukin-6, interleukin $1 \beta$ and so on) were recognized as active factors involved in the main biological functions of treatment, which implied that these were involved in the underlying mechanisms of strychnos nux-uomical on peripheral neuropathy.

Conclusions: In this study, a network pharmacology-based strategy integrating target prediction, network construction, GO and KEGG pathway enrichment analyses, was adopted to predict the targets of strychnos nux-uomical relevant to the treatment of peripheral neuropathy and to further explore the involved pharmacological mechanisms.

\section{Background}

The term peripheral neuropathy refers to any disorder of the peripheral nervous system including single and multiple (asymmetric) mononeuropathy, and symmetrical involvement of many nerves

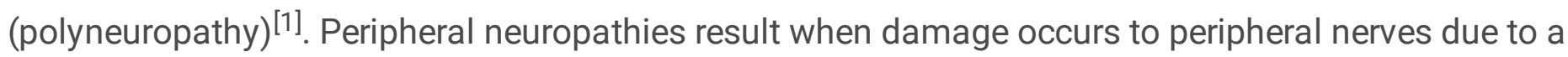
systemic disease, toxicant exposure or due to direct nerve injury ${ }^{[2]}$, result in loss of motor, sensory and autonomic functions in the denervated segments of the body, thus having strong impact in the quality of life of affected patients. Neuropathic pain is the most common clinical manifestation of peripheral neuropathy resulting from diabetes, chronic alcohol intake, certain cancers, vitamin B deficiency, infections, nerve-related diseases, toxins, and certain drugs ${ }^{[3]}$. Peripheral neuropathy occurs as a 
component of several common and many rare diseases ${ }^{[1]}$. Acquired neuropathies such as diabetic peripheral neuropathy, chemotherapy induced peripheral neuropathy and trauma/injury induced neuropathies occur as a result of enhanced oxidative stress and inflammation in peripheral neurons.

There are still no therapeutic agents available that can mitigate neuropathies in patients, and thus, more research is needed to identify molecular factors that can be effectively targeted.

Traditional chinese medicine (TCM) is featured as multiple targets and complex mechanisms of action and widely used in asian countries, especially China ${ }^{[4]}$, characterized by its satisfying therapeutic effects and minor side effects. Network pharmacology is an innovative method to study the mechanisms of drugs at the systemic level ${ }^{[5]}$, especially multi-target drugs. It encompasses bioinformatics, network analysis, and experimental approaches and integrates multiple sources of information ${ }^{[6]}$. Therefore, network approaches can accurately discriminate potential drug-target interactions ${ }^{[7]}$.

The strychnine tree (Strychnos nux-vomica L) (S.nux-vomica) belonging to family loganiaceae has been a very promising medication for certain disorders, used in TCM indications ${ }^{[8]}$ : protracted arthritis, rheumatoid arthralgia, numbness and paralysis, sequela of poliomyelitis, traumatic injury, boils and sores. Disease groups treated in TCM: nervous system ailments, locomotor, immune system ailments. However, the pharmacological mechanisms of S.nux-vomica are still unknown.

The current review is aimed at identifying the potential targets and molecular mechanisms underlying the effects of S.nux-vomica associated with various peripheral neuropathy by the network pharmacology strategy. First, we predicted the molecular targets of S.nux-vomica through chemical similarity analysis and pharmacophore model screening. Pathological targets were identified using various bioinformatics platforms. We then performed enrichment analysis according to GO and KEGG terms and constructed a drug-target-pathway network.

\section{Methods}

\section{Disease targets database building}

We collected peripheral neuropathy targets from the human gene database is a searchable, integrative database that provides comprehensive, user-friendly information on all annotated and predicted human genes, including genomic, transcriptomic, proteomic, genetic, clinical and functional information [9] "Peripheral neuropathy" as keywords to screen, there are 1231 targets, relevance score $>7$ were selected associated with peripheral neuropathy were screened through Gene-Cards as shown in Fig. 1. Finally, we matched the prediction of the targets of strychnos nux-uomical active ingredients and the retrieval of the related targets of peripheral neuropathy and chose the overlapping targets as the related targets of strychnos nux-uomical for the treatment of peripheral neuropathy. The targets were then processed by String to build a protein-protein interaction (PPI) network to analyze the interactions among these targets and screen the hub targets based on topology ${ }^{[10]}$. 


\section{Network construction method}

To collect the ingredients in strychnos nux-uomical, we used the traditional chinese medicine system pharmacology database ${ }^{[11]}$, a specialized pharmacological platform for TCM, to screen the active ingredients in strychnos nux-uomical by absorption, distribution, metabolism and excretion screening. In this process, the $\mathrm{OB}$ threshold was set as $30 \%$ and those ingredients with $\mathrm{OB} \geq 30 \%$ were selected as the active ingredients for the next step ${ }^{[12]}$. The $D L$ level of the compounds was set as 0.18 , as this is the selection criterion for "drug-like" compounds in traditional chinese herbs ${ }^{[13]}$. In this study, those ingredients with the number of related targes $>3 \square \mathrm{OB} \geq 30 \%$ and $\mathrm{DL} \geq 0.18$ were selected $^{[14]}$.

Active ingredients and related targets screening in strychnos nux-uomical, were then imported into cytoscape v3.2.1, an open software package project for visualizing, integrating, modeling and analyzing the interaction networks to generate compound-target network ${ }^{[15]}$.

The aim of our study used twice median degree values, was to screen the strychnos nux-uomical active ingredients and the related targets of peripheral neuropathy using cytoscape software merge tool to build a drug-target interaction network ${ }^{[16]}$.

To identify the hub nodes and essential proteins in the PPI network, the topological parameters of the node degree were calculated by Network Analyzer, and the three centralities (betweenness, closeness, and subgraph) were determined by the CytoNCA ${ }^{[17]}$ plugin to screen the core target of strychnos nux-uomical, used twice median degree values ${ }^{[16]}$.

\section{Enrichment Analyses}

The core targets associated with peripheral neuropathy treatment by strychnos nux-uomical were extracted from KEGG database and GO enrichment analysis, KEGG terms by panther database ${ }^{[18]}$ and constructed a drug-target-pathway network.

\section{Results}

\section{Active compounds and compound-target network networkin strychnos nux-uomical}

Retrieved from TCMSP, there were 13 active compounds were selected in strychnos nux-uomical. According to the active ingredient screening thresholds of $\mathrm{OB} \geq 30 \%, \mathrm{DL} \geq 0.18$ and the number of related targes $>3,13$ active ingredients were selected as shown in Table 1, 56 related targets were listed in Fig. 2. 
Table 1

The list of 13 active ingredients of strychnos nux-uomical

\begin{tabular}{|lll|}
\hline No. & ID & Compound \\
\hline 1 & MOL001040 & (2R)-5,7-dihydroxy-2-(4-hydroxyphenyl)chroman-4-one \\
\hline 2 & MOL001476 & (S)-Stylopine \\
\hline 3 & MOL003410 & Ziziphin_qt \\
\hline 4 & MOL003411 & Icaride A \\
\hline 6 & MOL003413 & Isostrychnine N-oxide (I) \\
\hline 7 & MOL003418 & Lokundjoside_qt \\
\hline 8 & MOL003432 & Vomicine \\
\hline 10 & MOL003436 & Isobrucine \\
\hline 11 & MOL003440 & Brucine N-oxide \\
\hline 12 & MOL000449 & Stigmasterol \\
\hline 13 & MOL000492 & (+)-catechin \\
\hline
\end{tabular}

\section{PPI Network Construction and Screening of Core Targets}

A total of 27 component core targets were screened used median degree values: cholinergic receptor muscarinic 2 (CHRM2), cholinergic receptor, muscarinic 1 (CHRM1), alpha-1D adrenergic receptor (ADRA1D), 5-hydroxytryptamine receptor 2A (HTR2A), alpha-1B adrenergic receptor (ADRA1B), nuclear receptor subfamily 3, group $C$, member 1 (NR3C1), solute carrier family 6 member 2 (SLC6A2), solute carrier family 6 member 3 (SLC6A3), solute carrier family 6 member 4 (SLC6A4), 5-hydroxytryptamine receptor 7 (HTR7), nuclear receptor coactivator 1 (NCOA1), nuclear receptor coactivator 1 (NCOA2), heat shock protein HSP 90-alpha (HSP90AA1), 5-hydroxytryptamine receptor 3A (HTR3A), mu-pioid receptor gene (OPRM1), alpha-2A adrenergic receptor (ADRA2A), androgen receptor (AR), catalase (CAT), progesterone receptor (PGR), opioid receptor delta 1 (OPRD1), monoamine oxidase $B$ (MAOB), monoamine oxidase $A$ (MAOA), nicotinic-acetylcholine receptor alpha 7 (CHRNA7), $\beta 1$-adrenergic receptor (ADRB1), $\beta 2$-adrenergic receptor (ADRB2), prostaglandin endoperoxide synthase2 (PTGS2), estrogen receptor alpha (ESR1). The disease core targets: RAC-alpha serine/threonine-protein kinase (AKT1), fibronectin 1(FN1), signal transducer and activator of transcription 3 (STAT3), tumor protein P53 (TP53), interleukin-10 (IL10), interleukin1 $\beta$ (IL1 $\beta$ ), vascular endothelial growth factor A (VEGFA), interleukin-6 (IL6), recombinant cytochrome C (CYCS), matrix metallopeptidase 9 (MMP9), insulin (INS), endothelial growth factor (EGF), tumor necrosis factor (TNF), ableson (ALB), amyloid precursor protein (APP), toll like receptor4 (TLR4), interleukin-8 (CXCL8), caspase-3 (CASP3). Analyzed by the Cytoscape plugin BisoGenet 
to generate the protein-protein interaction (PPI) network about Component-disease interactions (Fig. 3). We used the CytoHubba plugin in Cytoscape software according to twice median degree values to screen node component-disease interaction core target network, after screening, 72 core targets were obtained, as shown in Fig. 3.

\section{GO biological process and KEGG pathway enrichment analysis}

By enrichment analysis of the core target of strychnos nux-uomical in the PPI network, biological processes enrichment analysis and KEGG pathway enrichment analysis results in peripheral neuropathy shown in Fig. 4 and Fig. 5. The top five GO biological processes were mainly involved in cellular process, response to stimulus, biological regulation, metabolic process, localization. We selected 10 KEGG pathways for analysis.

The processes were, in the aspect of cellular process (GO:0009987): signal transduction (G0:0007165), cellular response to stimulus (G0:0051716) and cellular metabolic process (G0:0044237); in the aspect of response to stimulus(G0:0050896): response to chemical (G0:0042221), cellular response to stimulus (G0:0051716), response to stress (G0:0006950); in the aspect of biological regulation(G0:0065007): regulation of biological process (G0:0050789), regulation of molecular function (G0:0065009) and regulation of biological quality (G0:0065008); in the aspect of metabolic process (G0:0008152): biosynthetic process (G0:0009058) cellular metabolic process (G0:0044237) and organic substance metabolic process (GO:0071704); in the aspect of localization (G0:0051179): localization of cell (G0:0051674), establishment of localization (GO:0051234) and cellular localization (GO:0051641). The details are shown in Fig. 4. Based on these main aspects, a complex multi-path synergetic effect may be the cause of the effect of strychnos nux-uomical on peripheral neuropathy shown in Table 2.

The top ten KEGG pathways: inflammation mediated by chemokine and cytokine signaling pathway (P00031), CCKR signaling map (P06959), angiogenesis (P00005), gonadotropin-releasing hormone receptor pathway (P06664), apoptosis signaling pathway (P00006), p53 pathway feedback loops 2 (P04398), ras pathway (P04393), interleukin signaling pathway (P00036), VEGF signaling pathway (P00056) and T cell activation (P00053), as shown in Table 3. 
Table 2

Biological processes GO annotation analysis results (Top 5)

\begin{tabular}{|ll|}
\hline First Class & Second Class (Top 3) \\
\hline cellular process (GO:0009987) & signal transduction (GO:0007165) \\
& cellular response to stimulus (GO:0051716) \\
& cellular metabolic process (G0:0044237) \\
\hline response to stimulus (G0:0050896) & response to chemical (G0:0042221) \\
& cellular response to stimulus (GO:0051716) \\
& response to stress (GO:0006950) \\
\hline biological regulation (G0:0065007) & regulation of biological process (G0:0050789) \\
& regulation of molecular function (G0:0065009) \\
& regulation of biological quality (G0:0065008) \\
\hline metabolic process (GO:0008152) & biosynthetic process (GO:0009058) \\
& cellular metabolic process (GO:0044237) \\
& organic substance metabolic process (G0:0071704) \\
\hline localization (GO:0051179) & localization of cell (GO:0051674) \\
& establishment of localization (G0:0051234) \\
& cellular localization (GO:0051641) \\
\hline
\end{tabular}

Table 3

KEGG pathway enrichment analysis results (Top 10)

\section{Pathway}

Inflammation mediated by chemokine and cytokine signaling pathway (P00031)

CCKR signaling map (P06959)

Angiogenesis (P00005)

Gonadotropin-releasing hormone receptor pathway (P06664)

Apoptosis signaling pathway (P00006)

\section{Pathway}

p53 pathway feedback loops 2 (P04398)

Ras Pathway (P04393)

Interleukin signaling pathway (P00036)

VEGF signaling pathway (P00056)

T cell activation (P00053)

\section{Discussion}


There is an urgent need to develop new treatment strategies because of the growing crisis resulting from peripheral neuropathy and the poor efficacy of the existing approved drugs. We discovered that strychnos nux-uomical signifcantly alleviated the symptoms of peripheral neuropathy and believe that its impact may be related to its potential function. Hence, a network pharmacology strategy was used to reveal the mechanism underlying the effects of strychnos nux-uomical. The basic pathological changes of peripheral neuropathy include enhanced oxidative stress, inflammation and mitochondrial dysfunction in peripheral neurons $^{[19]}$.

Therapeutic targets and the signaling pathways in which they participate were explored by PPI network construction, and pathway enrichment analysis. Information from various online servers and databases was integrated. The results we obtained are very reliable, as they benefited from multiple information sources and different target identification methods.

Indicated by the KEGG pathway enrichment analysis, we proposed that strychnos nux-uomical plays a therapeutic role in the treatment of Peripheral neuropathy that is mediated by Inflammation mediated by chemokine and cytokine signaling pathway, p53 pathway feedback loops 2, CCKR signaling map, Ras Pathway, Angiogenesis, Interleukin signaling pathway, Gonadotropin-releasing hormone receptor pathway, VEGF signaling pathway, Apoptosis signaling pathway, T cell activation and so on.

\section{Inflammation mediated by chemokine and cytokine signaling pathway}

Inflammation has been indicated as a powerful driver of peripheral neuropathy. chemotherapy-induced increase in peripheral pro-inflammatory cytokines and a strong correlation with peripheral neuropathy, such as interleukin-6 (IL-6), tumor necrosis factor-a (TNFa), interleukin-1 $\beta$ (IL1 $\beta$ ), and interleukin-10 (IL-10) may play a role in pain ${ }^{[20]}$, IL $1 \beta^{[21]}$, which starts an immune response and leads to chronic inflammation.

This eventually results in apoptosis and bioenergetic dysfunction associated with DN. Glucose induced advanced glycation end products (AGE) and its further association with receptors for AGE also involved in activation of NF-KB heterodimer and thus releases proinflammatory mediators such as IL-6, TNF-a, cyclooxygenase-2 release and thus produces neuro inflammation ${ }^{[22]}$.

\section{p53 pathway feedback loops 2}

The p53 circuit communicates with the Wnt-beta-catenin, IGF-1-AKT, Rb-E2F, p38 MAP kinase, cyclin-cdk, p14/19 ARF pathways and the cyclin PP2A, and p73 gene products ${ }^{[23]}$. One of the most intriguing methods of activating the $\mathrm{p} 53$ protein in a cell results from the mutational inactivation of some tumor suppressor genes including retinoblastoma, and adenomatous polyposis coli or the mutational activation of some oncogenes such as ras and myc.

Cytochrome $c$ interacts with APAF-1 (a p53-regulated gene) to initiate a protease cascade, leading to the activation of caspase 9 and then caspase-3 followed by apoptosis. This is the intrinsic apoptotic 
pathway that is initiated by a number of stress signals that activate the p53 pathway. In addition to the intrinsic pathway, p53 regulates a series of genes that initiate the extrinsic apoptotic pathway (Fas ligand, killer Dr receptor), resulting in the caspase-8 and -3 activities and apoptosis ${ }^{[23]}$.

\section{Ras Pathway}

Ras homolog family (Rho, belong to Ras superfamily)/Rho-associated coiled-coil kinase (ROCK) signaling pathway widely exists in human and mammal cells, which is closely related to inhibition of repair after optic nerve damage ${ }^{[24]}$.

The Rho/ROCK/LIMK pathway is central for the mediation of repulsive environmental signals in the central nervous system. Inhibition of neurite outgrowth is counteracted by downregulation of ROCK2 and LIMK1. Three major biological effects can be specififically attributed to the knockdown of ROCK2: inhibition of apoptosis, attenuation of axonal degeneration and promotion of neurite outgrowth ${ }^{[25]}$.

\section{CCKR signaling map}

Gastrin and cholecystokinin receptor (CCKR) signaling events are indicated ${ }^{[26]}$, including proliferation, migration, differentiation, anti-apoptosis and inflammation. While central modules such as PKC, AKT1, Rho GTPase, MAP3K11, MAPK1/3 and AP1 are reportedly involved in all three cellular outcomes, other signaling mechanisms are more specific in their effect, e.g. the BCL-module signaling is only observed to be involved in apoptosis.

\section{Angiogenesis}

Vascular endothelial growth factor (VEGF) ${ }^{[27]}$ represents one of the main factors involved not only in angiogenesis and vasculogenesis but also in neuritogenesis. Modulating the local immune system to recruit cells to promote endogenous angiogenesis could have advantages, such as a greater degree of endothelial cell polarization. Furthermore, the immune system has a critical role in resolving inflammation following injury, where macrophages also have additional roles in this aspect of tissue regeneration.

\section{Gonadotropin-releasing hormone receptor pathway}

Gonadotropin-releasing hormone $(\mathrm{GnRH})$ is a decapeptide that acts not only as the principal hormone controlling puberty, but also as neuromodulatory hormone ${ }^{[28]}$. The current study addressed to the dorsal root ganglion(DRG)extended molecular profling towards gene expression analysis of genes known to be involved in the gonadotropin releasing hormone receptor and neuro infammatory (oxytocin/cytokines/chemokines) signaling pathways ${ }^{[29,30]}$. Serum levels of pro-inflammatory mediators such as IL-1 $\beta$, TNF- $\alpha$, and IL- 6 were significantly increased compared to healthy individuals within the entire study period,IL-1 $\beta$ plays a critical role in neuropathic pain, metabolic disorders, heart failure, and depression [31].

Growing evidence suggests that many representative growth factors, such as VEGF, insulin like growth factor (IGF), nerve growth factor (NGF), brain derived neurotrophic factor (BDNF), and fibroblast growth 
factor-2 (FGF2) have dual neurotrophic and angiogenic effects and were even named angioneurins ${ }^{[31]}$.

\section{Conclusions}

The potential targets and molecular mechanisms underlying the effects of strychnos nux-uomical against peripheral neuropathy mediated by chemokine and cytokine signaling pathway, p53 pathway feedback loops 2, CCKR signaling map, ras pathway, angiogenesis, interleukin signaling pathway, gonadotropin-releasing hormone receptor pathway, VEGF signaling pathway, apoptosis signaling pathway, $T$ cell activation in the treatment of peripheral neuropathy were systematically investigated by network pharmacology. This research provides a scientific basis for further elucidating the mechanism underlying the effects of Strychnos nux-uomical in the treatment of Peripheral neuropathy and suggests that the network pharmacology approach can be an effective tool to reveal the mechanisms of TCM. However, to improve the reliability of the results, further experimental experiments are needed to validate these results.

\section{Abbreviations}

OB: oral bioavailability; DL: drug likeness; GO: gene ontology; KEGG: kyoto encyclopedia of genes and genomes; TCM: traditional chinese medicine; PPI: protein-protein interaction; TCMSP: traditional chinese medicine system pharmacology platform; IL10: interleukin-10; IL1 $\beta$ : interleukin1 $\beta$; VEGFA: vascular endothelial growth factor A; IL6: interleukin-6; CYCS: recombinant cytochrome C; MMP9: matrix metallopeptidase 9; INS: insulin; EGF: endothelial growth factor; TNF: tumor necrosis factor; ALB: ableson; APP: amyloid precursor protein; toll like TLR4: receptor4; CXCL8: interleukin-8; CASP3: caspase-3;IL-6: interleukin-6; TNFa: tumor necrosis factor-a; IL1ß: interleukin-1ß; IL-10: interleukin-10; AGE: advanced glycation end products; ROCK: Rho-associated coiled-coil kinase; CCKR: gastrin and cholecystokinin receptor; VEGF: Vascular endothelial growth factor; GnRH: gonadotropin-releasing hormone; IGF: insulin like growth factor; NGF: nerve growth factor; BDNF: brain derived neurotrophic factor; FGF2: fibroblast growth factor-2.

\section{Declarations}

\section{Acknowledgements}

Not applicable.

\section{Authors' contributions}

Conceptualization, DS, S-JP and C-JF; methodology, validation and formal analysis, X-JJ, L-ZJ, ZY and J$\mathrm{KT}$; investigation $\mathrm{ZY}, \mathrm{ZN}, \mathrm{G}-\mathrm{JY}$ and $\mathrm{H}-\mathrm{ZR}$; data curation and writing the original draft preparation, $\mathrm{X}-\mathrm{JJ}$; writing, review, and editing, X-JJ and DS. All authors read and approved the final manuscript.

\section{Funding}


This study was supported by Zhejiang TCM Scientific Research Fund Project in 2021 (2021ZB092) and the Youth Project of the National Natural Science Foundation of China (81800138).

\section{Ethics approval and consent to participate}

Not applicable.

\section{Availability of data and materials}

The data used to support the findings of this study are available from the corresponding author upon request.

\section{Consent for publication}

The manuscript is approved by all authors for publication.

\section{Competing interests}

The authors declare that they have no competing interests.

\section{Author details}

Correspondence: Shu Deng, E-mail:dengshu2021@163.com

1. Department of Hematology, The First Affiliated Hospital of Zhejiang Chinese Medical University, Hangzhou 310000, China.

2. Zhejiang Chinese Medical University, Hangzhou 310000, China.

\section{References}

1. Martyn CN, Hughes RA. Epidemiology of peripheral neuropathy. J Neurol Neurosur Ps. 1997;62(4):310-8.

2. Ganesh Yerra V, Negi G, Sharma SS, Kumar A, et al. Potential therapeutic effects of the simultaneous targeting of the Nrf2 and NF-KB pathways in diabetic neuropathy. Redox Biol. 2013;1:394-7.

3. Panagiotis Z. Giustino V. Painful peripheral neuropathy and cancer. Pain Ther. 2017;6:115-6.

4. Li S, Xu TS. New development in traditional chinese medicine: symbolism-digit therapy as a special naturopathic treatment. Am J Chin Med. 2016;44(7):1311-23.

5. Hopkins AL. Network pharmacology: the next paradigm in drug discovery. Nat Chem Biol. 2008;4:682-90.

6. Musun P, Sa YP, Hae JL, et al. A systems-level analysis of mechanisms of platycodon grandifflorum based on a network pharmacological approach. Molecules. 2018;23(11):2841.

7. Boezio B, Audouze K, Ducrot P, et al. Network-based approaches in pharmacology. Mol Inform. 2017;36(10):170048. 
8. Chinese Pharmacopoeia Commission. Chinese Pharmacopoeia, Book 1. China Medical Science Press; 2015.

9. Rebhan M, ChalifaCaspi V, Prilusky J, et al. Genecards: integrating information about genes, proteins and diseases. Trends Gene. 1997;13(4):163.

10. Szklarczyk D, Franceschini A, Kuhn M, et al. The string database in 2011: functional interaction networks of proteins, globally integrated and scored. Nucleic Acids Res. 2011;39:561-8.

11. Ru JL, Li P, Wang JN, et al. TCMSP: a database of systems pharmacology for drug discovery from herbal medicines. J Cheminformatics. 2014; 6 .

12. Xu X, Zhang W, Huang $C$, et al. A novel chemometric method for the prediction of human oral bioavailability. Int J Mol Sci. 2012;13(6):6964-82.

13. Tao W, Xu X, Wang X, et al. Network pharmacology-based prediction of the active ingredients and potential targets of chinese herbal radix curcumae formula for application to cardiovascular disease. J Ethnopharmacol. 2013;145(1):1-10.

14. Assenov Y, Ramirez F, Schelhorn SE, et al. Computing topological parameters of biological networks. Bioinformatics. 2008;24(2):282-4.

15. Smoot ME, Ono K, Ruscheinski J, et al. Cytoscape 2.8: new features for data integration and network visualization. Bioinformatics. 2011;27(3):431-2.

16. Wang S, Wang H, Lu Y. Tianfoshen oral liquid: a CFDA approved clinical traditional chinese medicine, normalizes major cellular pathways disordered during colorectal carcinogenesis. Oncotarget. 2017;8(9):14549-69.

17. Chin $\mathrm{CH}$, Chen $\mathrm{SH}, \mathrm{Wu} \mathrm{HH}$, et al. Cytohubba: identifying hub objects and sub-networks from complex interactome. Bmc Syst Biol. 2014;8:11.

18. Mi H, Muruganujan A, Thomas PD. Panther in 2013: modeling the evolution of gene function, and other gene attributes, in the context of phylogenetic trees. Nucleic Acids Res. 2013;41(D1):D377-86.

19. Areti A, Veera GY, Komirishetty P, et al. Potential therapeutic benefits of maintaining mitochondrial health in peripheral neuropathies. Curr Neuropharmacol. 2016;14(6):593-609.

20. Brandolini L, d'Angelo $M$, Antonosante A, et al. Chemokine signaling in chemotherapy-induced neuropathic pain. Int J Mol Sci. 2019;20(12):2904.

21. Zhang Q, Raoof M, Chen $Y$, et al. Circulating mitochondrial DAMPs cause inflammatory responses to injury. Nature. 2010;464(7285):104-7.

22. Aparna A, Veera GY, Komirishetty P, et al. Potential therapeutic benefits of maintaining mitochondrial health in peripheral neuropathies. Curr Neuropharmacol. 2016;14(6):593-609.

23. Harris SL, Levine AJ. The p53 pathway: positive and negative feedback loops. Oncogene. 2005;24(17):2899-908.

24. Feng YB, LoGrasso PV, Defert O, et al. Rho kinase (ROCK)inhibitors and their therapeutic potential. J Med Chem. 2016;59(6):2269-300. 
25. Koch JC, Tonges L, Barski E, et al. Rock2 is a major regulator of axonal degeneration, neuronal death and axonal regeneration in the CNS. Cell Death Dis. 2014;5:e1225.

26. Tripathi S, Flobak A, Chawla K, et al. The gastrin and cholecystokinin receptors mediated signaling network: a scaffold for data analysis and new hypotheses on regulatory mechanisms. BMC Syst Biol. 2015;9:40.

27. Muratori L, Sara G, Fregnan F, et al. Evaluation of vascular endothelial growth factor (VEGF) and its family member expression after peripheral nerve regeneration and denervation. Anat Rec. 2018;301(10):1646-56.

28. Kathleen E, Whitlock. The loss of scents: do defects in olfactory sensory neuron development underlie human disease? Birth Defects Res C. 2015;105(2):114-25.

29. Gravius N, Chaudhry SR, Muhammad S, et al. Selective L4 dorsal root ganglion stimulation evokes pain relief and changes of infammatory markers: part I profling of saliva and serum molecular patterns. Neuromodulation. 2019;22(1):44-52.

30. Kinfe TM, Maria A, Chakravarthy KV, et al. Unilateral L4-dorsal root ganglion stimulation evokes pain relief in chronic neuropathic postsurgical knee pain and changes of inflammatory markers: part II whole transcriptome profiling. J Transl Med. 2019;17:205.

31. Han JW, Dabin C, Lee MY, et al. Bone marrow-derived mesenchymal stem cells improve diabetic neuropathy by direct modulation of both angiogenesis and myelination in peripheral nerves. Cell Transplant. 2016;25(2):313-26.

\section{Figures}




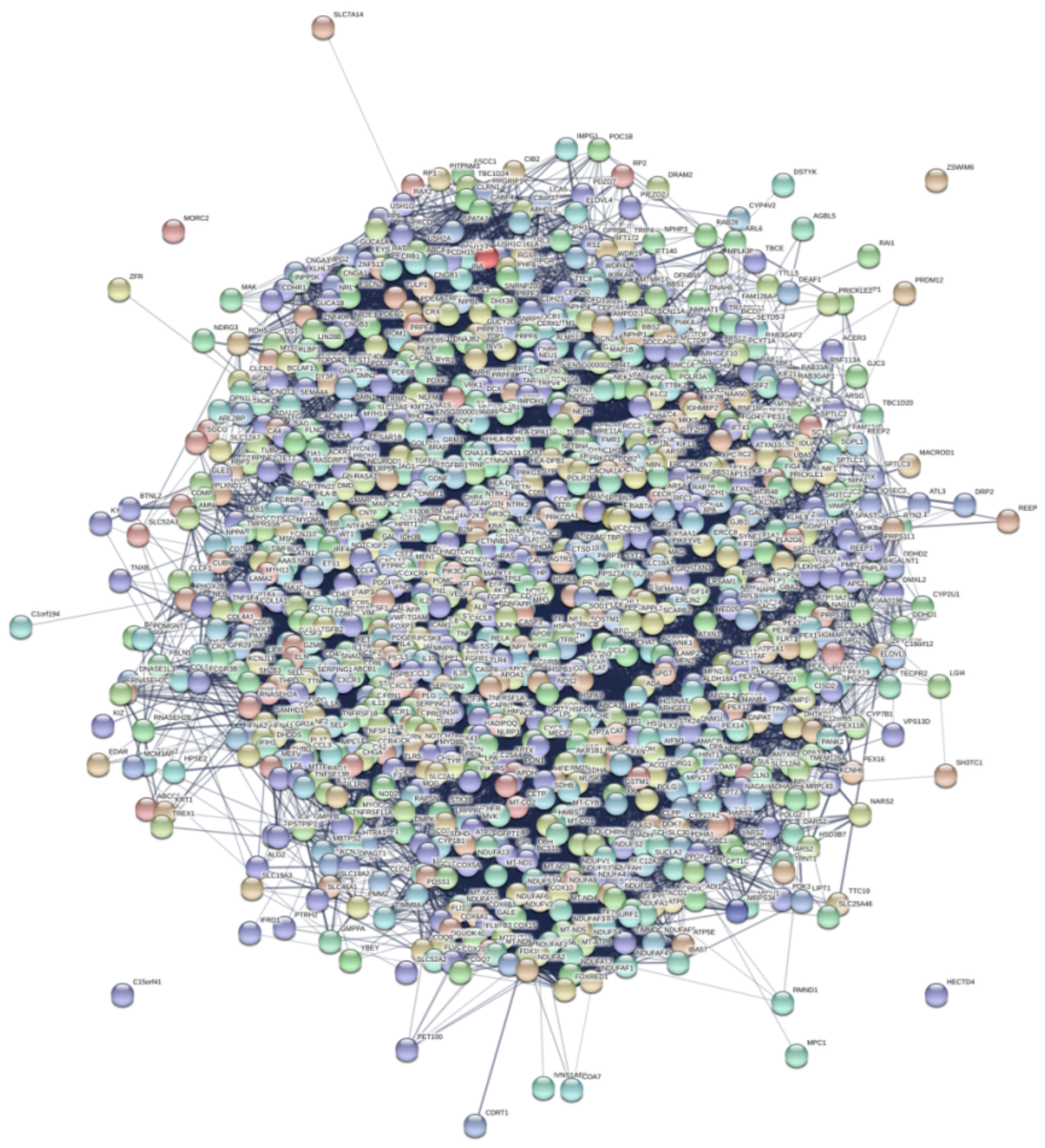

Figure 1

Targets network of peripheral neuropathy 


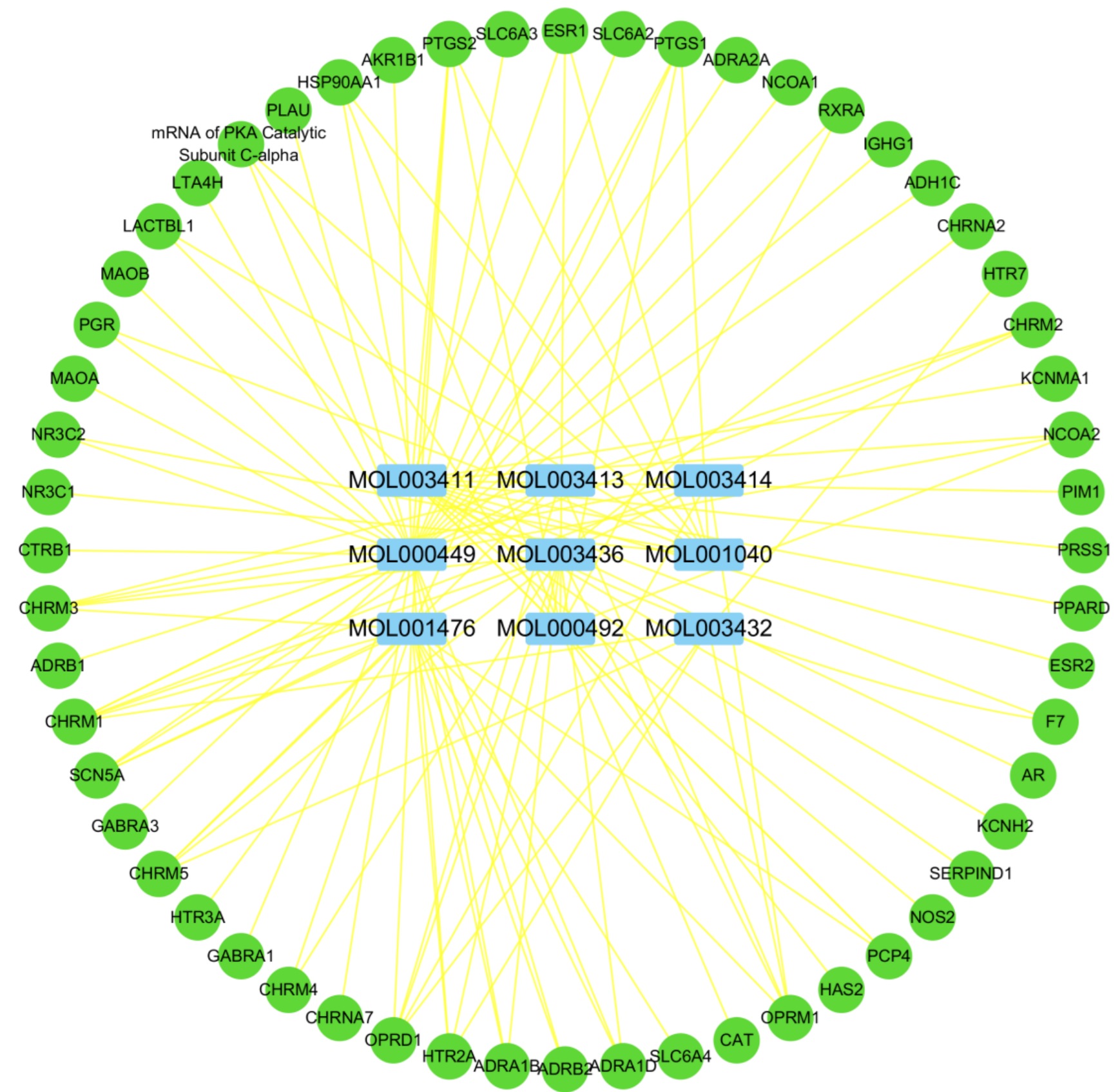

Figure 2

The components-target network in strychnos nux-uomical. Blue and green nodes denote the compounds and targets. 

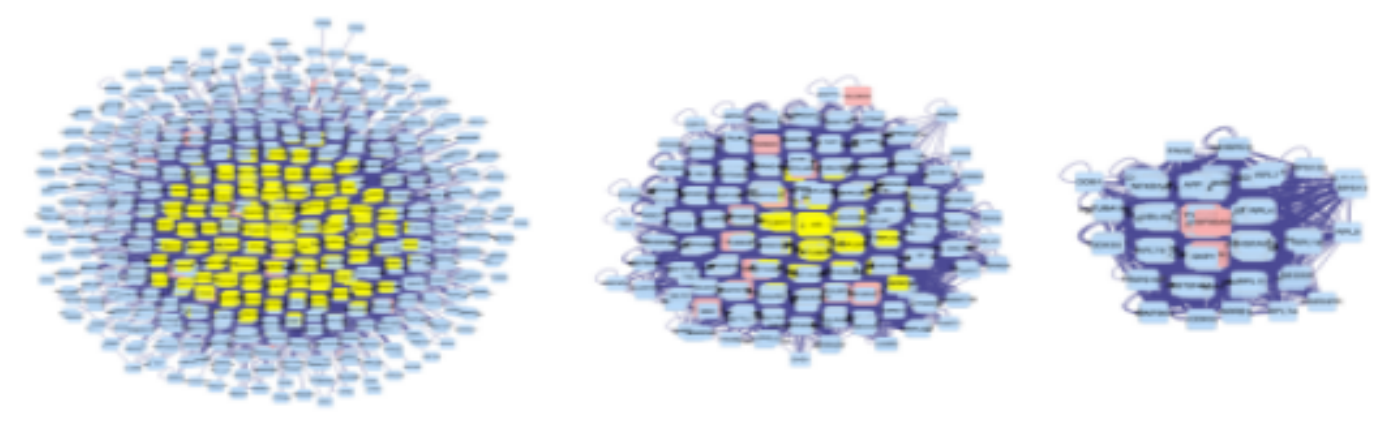

Figure 3

PPI Network Construction and Screening of Potential Targets
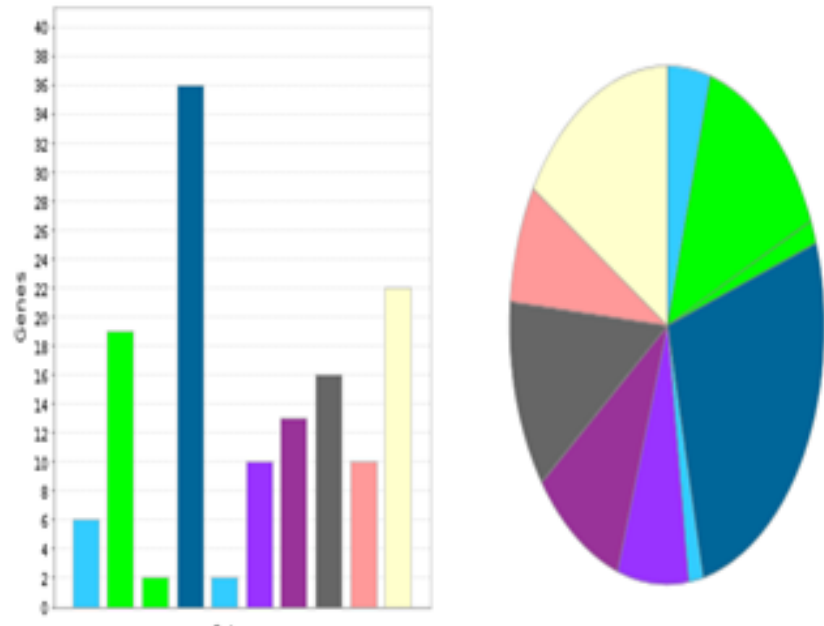

I belogial aftesion (co:0022610)

- balogid requacon (C0:0065007)

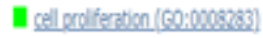

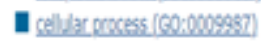

II develoomental cocoess (C0:0032502)

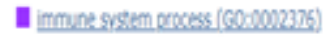

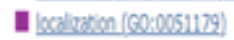

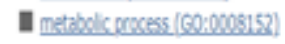

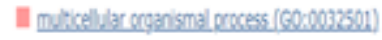

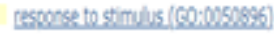

\section{Figure 4}

Biological processes $\mathrm{GO}$ annotation analysis results

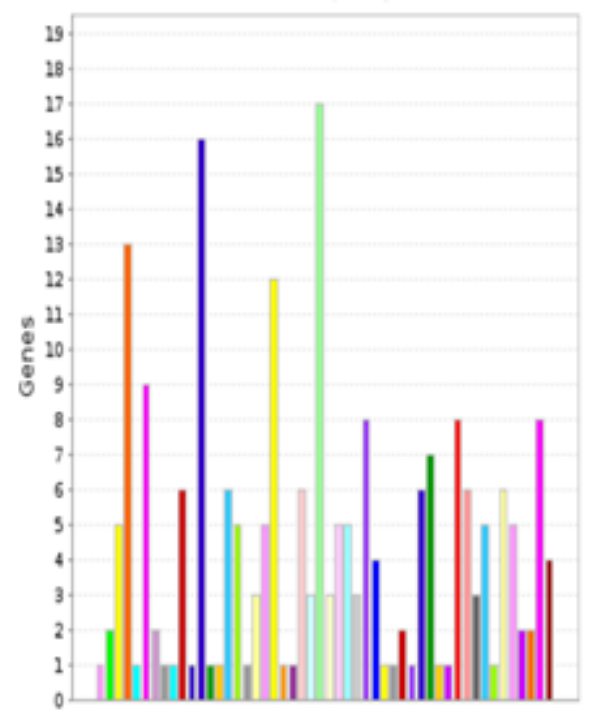

Category
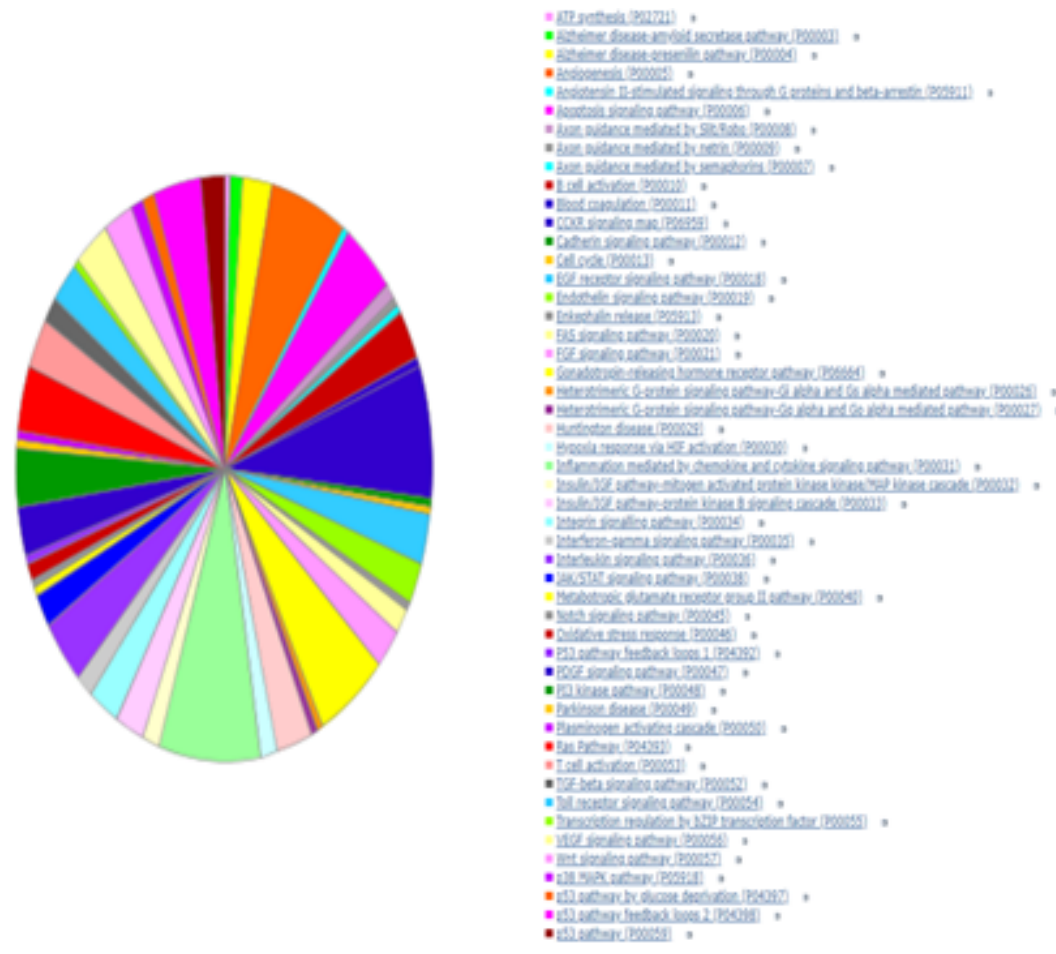
Figure 5

KEGG pathway enrichment analysis results 